\title{
Knowledge Driven Phenotyping
}

\author{
Honghan WU a,c,1, Minhong WANG ${ }^{\text {a }}$, Qianyi ZENG ${ }^{\text {a }}$, Wenjun CHEN $^{\mathrm{a}}$, \\ Jeff Z. PAN ${ }^{\mathrm{b}}$, Cathie SUDLOW ${ }^{\mathrm{a}, \mathrm{c}}$ and Dave ROBERTSON ${ }^{\mathrm{a}, \mathrm{c}}$ \\ ${ }^{\text {a }}$ The University of Edinburgh \\ ${ }^{\mathrm{b}}$ The University of Aberdeen \\ ${ }^{\mathrm{c}}$ Health Data Research, University of Edinburgh, United Kingdom
}

\begin{abstract}
Extracting patient phenotypes from routinely collected health data (such as Electronic Health Records) requires translating clinically-sound phenotype definitions into queries/computations executable on the underlying data sources by clinical researchers. This requires significant knowledge and skills to deal with heterogeneous and often imperfect data. Translations are time-consuming, error-prone and, most importantly, hard to share and reproduce across different settings. This paper proposes a knowledge driven framework that (1) decouples the specification of phenotype semantics from underlying data sources; (2) can automatically populate and conduct phenotype computations on heterogeneous data spaces. We report preliminary results of deploying this framework on five Scottish health datasets.
\end{abstract}

Keywords. health data, phenotype computation, data integration, ontology

\section{Introduction}

Big data analytics in healthcare has great potential to reveal deep insights from health data, which would extend our knowledge boundary in medicine and improve quality of health service [1]. However, it is very challenging to make sense of distributed and heterogeneous health data. The current reality is that most data is stored in different local communities, which means they are maintained locally and stored in inconsistent formats and languages. A key technical challenge haunting almost all data-driven clinical studies is to extract or compute accurate patients' phenotypes (traits of symptoms, diseases, medications or biochemistry test results) from such a fragmented data space.

Figure 1 (Current Practice section on the left-hand side) illustrates a typical procedure of computing phenotypes from heterogeneous data sources. The first step, and scientifically the most important, is to specify what constitutes a phenotype using clinical knowledge/terminologies that the clinical research community are familiar with, e.g. using languages in laboratory, medicine, oncology and genetics etc. These specified phenotypes must be computed (extracted or inferred) from the underlying health data. Their computation (steps $2 \& 3$ ) requires (each time) significant human effort to understand database details, good data science skills to do the querying and data manipulating, and caution \& patience to deal with data incompleteness/inconsistencies. The translations from phenotype specifications into computations are hard to verify and debug, which

\footnotetext{
${ }^{1}$ Corresponding Author: 9 Little France Road, Edinburgh EH16 4UX, UK; E-mail: honghan.wu@ed.ac.uk.
} 


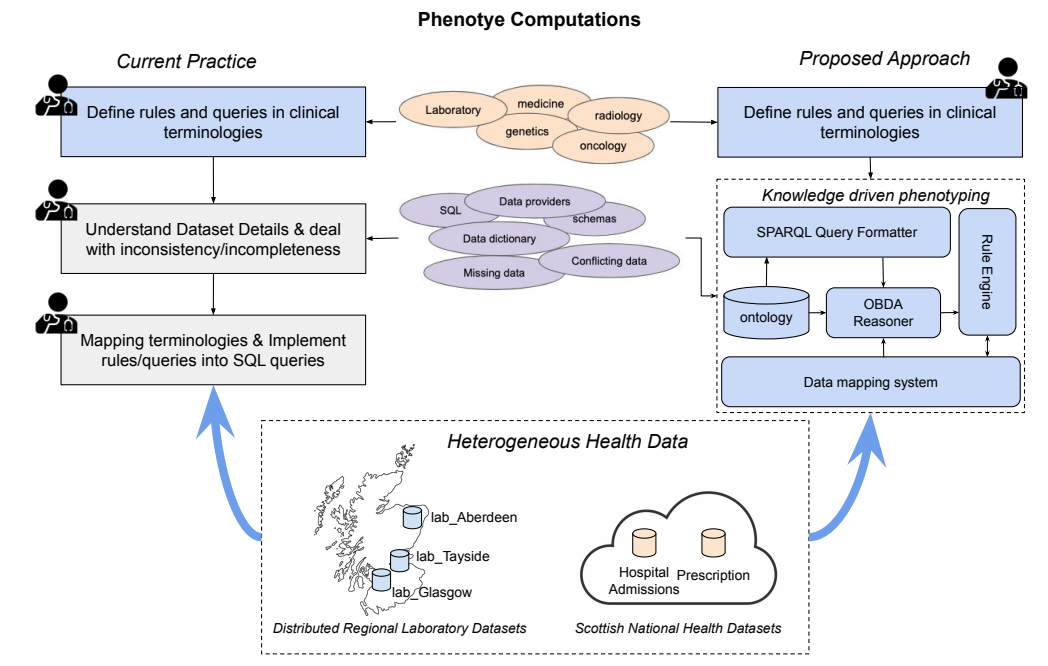

Figure 1. System architecture. A knowledge driven phenotyping framework that frees clinical researchers from time-consuming and error-prone translations from clinical rules to database-level queries.

poses threats to the understandability and credibility of data-driven studies. In addition, due to the sensitive nature of health data, such translations cannot be made public, which significantly impedes the reusability and reproducibility of clinical researches. In this paper, we propose a knowledge driven framework to tackle these challenges.

\section{Method}

The main aim of this study is to realise a clinical data science framework that makes the underlying data sources transparent to phenotype computations. Researchers only need to specify the "meanings" of their phenotypes using their familiar terminologies and the actual computations are automatically populated for and executed on data sources.

For example, to retrieve a cohort of patients with Type 2 Diabetes in Scotland, such a framework would only require researchers to provide an assertion like Type2_Diabetes_Patient (?x) without them knowing that the national Hospital Admissions is the underlying database, which is a Microsoft SQL Server managed by eDRIS team (https: //www . isdscotland .org/Products-and-Services/eDRIS/) (therefore, they have the data dictionary) and uses both ICD 9 and 10 for diagnosis coding due to legacy data. To realise that, the key is to decouple the formalisation of phenotype semantics and the management/technical details of underlying data sources. We propose an architecture (see Figure 1) to implement such a decoupling, which is roughly composed of two aspects: phenotype formalisation framework (for specifying phenotype semantics) and ontology/rule based data access (for automated phenotype computing on data sources).

\subsection{Phenotype Formalisation Framework}

Given a phenotype such as Type2_Diabetes_Patient, the computer has to understand its semantics (i.e., computer understandable meanings) so that the right computations and 
queries can be populated and executed on the underlying data sources. The formalisation framework has three components as follows.

- A database independent phenotype formalisation using Semantic Web knowledge representation technologies. Specifically, we use DL Lite [2] ontologies and Semantic Web Rule Language (SWRL) [3] queries to define phenotype semantics.

- A core phenotype ontology serving as the base vocabulary linking to standard clinical terminologies available at BioPortal [4] (e.g., SNOMED CT, ICD10).

- A query formatter that generates ontology queries (SPARQL [5]) from an user interface. The formatter can automatically translate phenotype definitions between standard terminologies (e.g., READ to SNOMED CT) via UMLS [6].

We use Type 2 Diabetes as a running example to illustrate phenotype specifications in this framework.

Phenotype Definition using Ontology The following equation defines a DL-Lite axiom for computing the phenotype of Type 2 Diabetes, meaning anyone who had a diagnosis of ICD10 code E11 is a Type 2 Diabete Patient.

$$
\text { icd10:E11(?x) } \Rightarrow \text { Type2_Diabetes_Patient }(? x)
$$

Standard Terminology Inclusion and Subsumption Inference The prefix icd10: indicates that E11 is a concept of ICD10 ontology, which is then incorporated in the phenotype computing. This automatically includes all the semantics defined in ICD10, such as icd10:E11.2 巨icd10:E11. Based on DL-Lite logic, such a class subsumption relation will be automatically combined with Axiom (1) to infer that icd10:E11.2 Type2_Diabetes_Patient. Similarly, instances of all other sub-classes of E11 are inferred as instances of Type2_Diabetes_Patient.

Rule for Dealing with Incompleteness If a researcher worries the diagnosis data might miss some Type 2 Diabetics, she might think of certain drug uses which could infer the disease phenotype with acceptable reliability. Rule (2) infers that a patient prescribed Sulphonylurea $S R$ is potentially a Type 2 Diabetes patient.

$$
\text { hasPrescription }(? x, ? p) \wedge \text { prescribedDrug }(? p, ? d)
$$

$\wedge$ drugName $(? d$, “Sulphonylurea SR”) $\Rightarrow$ Drug_Inferred_Type2_Diabetes $(? x)$

Rule for Dealing with Inconsistency Erroneous and inconsistent facts are almost inevitable when using clinical data for research. For example, clinically, it is very unlikely a person is both Type 1 and Type 2 Diabetic. The rule and axiom in (3) define a class of DiabetesConflictPatient as a sub-class of PotentialCodingErrorPatient in the core ontology, which detects potential coding errors in health data.

$$
\begin{array}{r}
\text { ICD10:E11(?x) } \wedge \text { ICD10:E10 }(? x) \Rightarrow \text { DiabetesConflictPatient }(? x) . \\
\text { DiabetesConflictPatient } \sqsubseteq \text { PotentialCodingErrorPatient }
\end{array}
$$




\subsection{Ontology Based \& Rule Driven Data Access}

To automatically compute the above formalised phenotypes on actual data, we adopt ontology based data access (OBDA) techniques [7]. The OBDA system in our framework (on the right of Figure 1) is composed of three components: an ontology for specifying user queries, a data mapping system for translating ontology predicates into relational database schema/constructs and an OBDA reasoner conducting the translation and optimisation, for which we use an open source OBDA reasoner - OnTop [8].

Phenotype computations are heavily rule driven for two reasons: (1) most phenotyping semantic constraints are hard to represent using OWL Lite constructs (e.g., the rules described previously); (2) the semantics of most phenotypes are not fixed - they either change with research focuses or different researchers might have different opinions about certain rules related to a phenotype. Therefore, comparing to ontological formalisation, phenotype semantics needs more customisable/flexible specification approaches like rule languages. However, the current official release of OnTop does not support SWRL rules.

For this reason, in our framework, we minimise the core ontology. A core mapping from this ontology to the underlying data sources is manually created to initiate the data mapping system. To support rule-driven phenotype specification, a rule engine is implemented. The engine can automatically convert SWRL rules into new data mappings by utilising the core mapping and the ontology semantics. The populated mappings are then merged with the core mapping in the rule engine, which will be loaded in the OBDA component on the fly to do phenotype computations.

\section{Deployment and Evaluation}

This study is supported by Health Data Research UK (https://www.hdruk.ac.uk/ projects/graph-based-data-federation-for-healthcare-data-science/) as an exemplar to create a federation of distributed health data in Scotland. The above described framework has been deployed on 5 synthetic data sets (as shown at the bottom of Figure 1) generated using BadMedicine (https://github.com/HicServices/ BadMedicine), which represents data/schema characteristics learnt from real data.

Due to space limitations, we put the full benchmark and evaluation details on a Github page: https://github.com/Honghan/KGPhenotyping/tree/master/ evaluation. In Table 1, we compares the ontology queries (SPARQL) and SQL queries for three exemplar phenotype definitions (in simplified forms; actual queries are at https://bit.ly/2oZK5rK).

1. (Terminology Mapping) The first phenotype is defined using SNOMED CT (321949006) to specify a prescription. Scottish Prescription data is using BnfCode for drug identifiers. Our framework can take the SNOMED CT code as the input and translate it into BnfCode automatically, while in Current Practice the user has to understand that BnfCode is used in the data and need to get equivalent BnfCode (0403030E0AAAAAA) for the given code.

2. (Inference) The second phenotype is to get patients with Type 2 Diabetes defined as ICD10 E11 and its sub-codes. However, in Scottish Hospital Admissions dataset, both ICD10 and ICD9 are used. Therefore, using SQL, it requires us to give an exhaustive list of codes ( 20 codes). In our framework, the ICD9/10 hier- 


\begin{tabular}{|c|c|c|}
\hline Phenotype Definition & $\begin{array}{l}\text { SPARQL } \\
\end{array}$ & SQL \\
\hline $\begin{array}{l}\text { patients prescribed Fluoxetine (SNOMED CT } \\
\text { code: } 321949006 \text { ) }\end{array}$ & $\begin{array}{l}? x \text { hasPrescription } ? p \\
? p \text { a snomed:321949006 }\end{array}$ & $\begin{array}{l}\text { select CHI from Prescription } \\
\text { where BnfCode }=\text { '0403030EOAAAAAA', }\end{array}$ \\
\hline $\begin{array}{l}\text { patients diagnosed as Type } 2 \text { Diabetes (ICD10 } \\
\text { E11 and its sub-codes) }\end{array}$ & ?x a icd10:E11. & $\begin{array}{l}\text { select CHI from Admissions where maincondition like } \\
\text { 'E11\%' or maincondition in ('-25000', '-25002', }\end{array}$ \\
\hline $\begin{array}{l}\text { patients with potential Diabetes coding errors - } \\
\text { diagnosed as both Type } 2 \text { Diabetes (ICD10 E11 } \\
\text { and its sub-codes) and Type } 1 \text { Diabetes (ICD10 } \\
\text { E10 and its sub-codes) }\end{array}$ & $\begin{array}{l}? x \text { a Diabetes ConflictPa- } \\
\text { tient } .\end{array}$ & $\begin{array}{l}\text { select h1.CHI from HospitalAdmissions hl, Hospital- } \\
\text { Admissions } \mathrm{h} 2 \text { where (h1.maincondition like 'E11\%' } \\
\text { or h1.maincondition in ('-25000', '-25002', ..)) and } \\
\text { (h2.maincondition like 'E10\%' or h1.maincondition } \\
\text { in ('-25001', '-25003', ..)) and h1.CHI = h2.CHI }\end{array}$ \\
\hline
\end{tabular}

Table 1. SPARQL vs. SQL queries for the three phenotype definitions on 5 Scottish Health Datasets

archies and their mapping are automatically utilised for doing inferences. Therefore, only a simple icd10:E11 predicate will be sufficient for the computation.

3. (Inconsistency Checking) The third phenotype is to detect potential Diabetes coding errors in the data (Type 1 and 2 are exclusive). With Rule 3, our framework only needs one predicate DiabetesConflictPatient, while SQL will need around 40 codes and a inner join operation on the Hospital Admission table.

\section{Conclusion}

To overcome obscure phenotype computation, which makes experiments difficult to understand and reproduce, we developed a new framework to allow clinical researchers to formalise phenotype semantics independently to the data and, more importantly, in a computer understandable way so that its computation can be automated on the underlying data sources. We implemented a knowledge-driven (based on ontologies and rule languages) approach to define an interlingua in which practitioners can represent the phenotype semantics they want to use and automatically translates this to computations as database queries on participating data sources.

\section{References}

[1] Raghupathi, W. and Raghupathi, V., 2014. Big data analytics in healthcare: promise and potential. Health information science and systems, 2(1), p.3.

[2] Calvanese, D., De Giacomo, G., Lembo, D., Lenzerini, M. and Rosati, R., 2007. Tractable reasoning and efficient query answering in description logics: The DL-Lite family. Journal of Automated reasoning, 39(3), pp.385-429.

[3] Horrocks, I., Patel-Schneider, P.F., Boley, H., Tabet, S., Grosof, B. and Dean, M., 2004. SWRL: A semantic web rule language combining OWL and RuleML. W3C Member submission, 21(79), pp.1-31.

[4] Noy, N.F., Shah, N.H., Whetzel, P.L., Dai, B., Dorf, M., Griffith, N., Jonquet, C., Rubin, D.L., Storey, M.A., Chute, C.G. and Musen, M.A., 2009. BioPortal: ontologies and integrated data resources at the click of a mouse. Nucleic acids research, 37(suppl_2), pp.W170-W173.

[5] The W3C SPARQL Working Group. SPARQL 1.1 Overview - https://www.w3.org/TR/sparql11overview/. W3C Recommendation 21 March 2013.

[6] Bodenreider, O., 2004. The unified medical language system (UMLS): integrating biomedical terminology. Nucleic acids research, 32(suppl_1), pp.D267-D270.

[7] Xiao, G., Calvanese, D., Kontchakov, R., Lembo, D., Poggi, A., Rosati, R. and Zakharyaschev, M., 2018, July. Ontology-based data access: A survey. IJCAI.

[8] Calvanese, D., Cogrel, B., Komla-Ebri, S., Kontchakov, R., Lanti, D., Rezk, M., Rodriguez-Muro, M. and Xiao, G., 2017. Ontop: Answering SPARQL queries over relational databases. Semantic Web, 8(3), pp.471-487. 